\title{
Exponential Chebyshev Approximation on Finite Subsets of $[0,1]$
}

\author{
By Bernard H. Rosman
}

\begin{abstract}
In this note the convergence of best exponential Chebyshev approximation on finite subsets of $[0,1]$ to a best approximation on the interval is proved when the function to be approximated is continuous and when the union of the finite subsets is dense in $[0,1]$.
\end{abstract}

1. Introduction. In this note we study the convergence of best exponential Chebyshev approximation on finite subsets of $[0,1]$ to a best approximation on the interval. This problem has been considered for linear approximation [1] and, recently, for generalized rational approximation in [2].

Let $X_{r}$ be a set of $r$ distinct points in [0,1], containing the endpoints (a common computational situation). We assume that the sequence of subsets $\left\{X_{r}\right\}$ fills up the interval in the sense that, given $x \in[0,1]$, there is an $x_{r} \in X_{r}$ such that $\left\{x_{r}\right\} \rightarrow x$.

Following Rice [3, Chapter 8], we approximate $f \in C([0,1])$ by exponential functions of the form

$$
E(A, x)=\sum_{i=1}^{k}\left(\sum_{j=0}^{m_{i}} p_{i j} x^{i}\right) e^{t_{i x}},
$$

where $\left|p_{i j}\right|<\infty,\left|t_{i}\right|<\infty$ and $\sum_{i=1}^{k}\left(m_{i}+1\right) \leqq n, n$ a fixed positive integer.

For each set $X_{r}$, we define the usual seminorm on $C([0,1])$, corresponding to $X_{r}$, by

$$
\|f\|_{x_{r}}=\sup _{x \in X_{r}}|f(x)|
$$

for all $f$ in $C([0,1])$. We denote by $E\left(A_{r}, x\right)$ and $E_{r}(x)$ the best approximation to $f(x)$ on $X_{r}$, i.e. the best approximation to $f$ with respect to the seminorm corresponding to $X_{r}$. Norm signs without subscripts denote the usual Chebyshev norm.

It is known [3] that best approximation need not exist on finite point sets. However, we assume existence, a reasonable assumption in many computational situations. Moreover, Rice solves a special case of this problem through the use of pseudo functions [3, pp. 65-69]. An extension of this technique to handle general exponential approximation is under investigation.

2. The Convergence Theorem. The main result of this note is

THEOREM 1. Let $E^{*}$ be a best exponential approximation to $f$ on $[0,1]$. Then

$$
\left\|f-E_{r}\right\|_{x_{r}} \rightarrow\left\|f-E^{*}\right\| \text {. }
$$

Received January 22, 1970, revised September 9, 1970.

AMS 1970 subject classifications. Primary 41A30, 41A50; Secondary 65D10.

Key words and phrases. Chebyshev approximation, exponential functions, varisolvence, pointwise convergence, uniform convergence. 
Proof. First, suppose that $\left\{\left\|E_{r}\right\|\right\}$ is a bounded sequence. Then by a theorem in Rice [3, Chapter 8], $\left\{E_{r}(x)\right\}$, or a subsequence thereof, converges pointwise to a possibly discontinuous function

$$
\begin{aligned}
E(x) & =E_{0}(x), & & 0<x<1, \\
& =e_{0}, & & x=0, \\
& =e_{1}, & & x=1,
\end{aligned}
$$

where $E_{0}(x)$ is an exponential of form (1). We claim that $E_{0}$ is a best approximation to $f$ on $[0,1]$. For if not, there exists a point $x$ such that $\left|f(x)-E_{0}(x)\right|>\left\|f-E^{*}\right\|$. By continuity, we may assume that $x \in[\delta, 1-\delta]$ where $\delta>0$ is sufficiently small. Let $\left\{x_{r}\right\} \rightarrow x$ with $x_{r} \in X_{r}, \epsilon>0$, and let $y_{1}, \cdots, y_{n}$ be in $[\delta, 1-\delta]$. By the definition of varisolvence, there is a $\delta(\epsilon)$ such that the inequality $\left|Y_{i}-E_{0}\left(y_{i}\right)\right|<\delta(\epsilon)$ implies that there is a function $E(A, y)$ satisfying $E\left(A, y_{i}\right)=Y_{i}$ and $\mid E(A, y)$ $E_{0}(y) \mid<\epsilon$. But since $E_{r} \rightarrow E_{0}$ pointwise on $[\delta, 1-\delta]$, for $r$ large enough, $\mid E_{r}\left(y_{i}\right)-$ $E_{0}\left(y_{i}\right) \mid<\delta(\epsilon)$. Hence, there are functions $\left\{E\left(A_{r}, y\right)\right\}$ such that $E\left(A_{r}, y_{i}\right)=E_{r}\left(y_{i}\right)$. But since these interpolating functions are unique ( $m$ is the degree of solvence), $E\left(A_{r}, y\right)=E_{r}(y)$ for all $y$. Therefore, $\left|E_{r}(y)-E_{0}(y)\right|<\epsilon$ for $y$ in $[\delta, 1-\delta]$. Therefore, by a standard inequality, $\left|f\left(x_{r}\right)-E_{r}\left(x_{r}\right)\right| \rightarrow\left|f(x)-E_{0}(x)\right|$. Hence, for sufficiently large $r,\left|f\left(x_{r}\right)-E_{r}\left(x_{r}\right)\right|>\left\|f-E^{*}\right\|$. This contradicts the fact that $E_{r}$ is a best approximation on $X_{r}$ and hence $E_{0}$ is a best approximation.

Suppose now that $\left\{\left\|E_{r}\right\|\right\}$ is an unbounded sequence. Following Dunham [2], define $B_{r}(x)=E_{r}(x) /\left\|E_{r}\right\|$. Then $\left\{\left\|B_{r}\right\|\right\}$ is a bounded sequence and, by the aforementioned result of Rice, we may assume that $\left\{B_{r}(x)\right\}$ converges pointwise to a function of the form

$$
\begin{aligned}
B(x) & =B_{0}(x), & & 0<x<1, \\
& =b_{0}, & & x=0, \\
& =b_{1}, & & x=1,
\end{aligned}
$$

where $B_{0}(x)$ is an exponential of form (1). Now, assume that $b_{0}=B_{0}(0)$ and $b_{1}=B_{0}(1)$. The $B(x)$ is an exponential and, using varisolvence as before, it follows that $\left\{B_{r}\right\}$ converges uniformly to $B$. Since $\left\|B_{r}\right\|=1$, there exists $y \in[0,1]$ and a neighborhood $N$ of $y$ such that $m=\inf _{x \in N} B(x)>0$ and $B_{r}(x)>m / 2$ for $r$ sufficiently large. Hence, $\inf _{x \in N} E_{r}(x) \rightarrow \infty$ as $r \rightarrow \infty$ and, for large enough $r$, there exists $x_{r} \in X_{r}$ such that $E_{r}\left(x_{r}\right)>2\|f\|$. This contradicts $E_{r}$ being a best approximation to $f$ on $X_{r}$ since then

$$
\left\|f-E_{r}\right\|_{X_{r}}>\|f\| \geqq\|f\|_{X_{r}}=\|f-0\|_{X_{r}} .
$$

It remains to consider the case where $B(x)$ has an endpoint discontinuity. Assume without loss of generality that $B(x)=B_{0}(x), 0<x \leqq 1$, and $b_{0}>B_{0}(0)$. If $B_{0} \neq \equiv 0$, there exists $y \in[\delta, 1-\delta], \delta>0$, such that $B_{0}(y) \neq 0$ and $\left\{B_{r}(y)\right\} \rightarrow B_{0}(y)$. Using the varisolvence of $B_{0}$ on this interval, we again contradict $E_{r}$ being best on $X_{r}$. If $B_{0} \equiv 0$, we reach the same contradiction on $E_{r}$ since then $\left\{E_{r}(0)\right\}$ is unbounded. This concludes the proof.

If we cannot assume that the endpoints are in $X_{r}$, the proof goes through without change except for the last case. If $B_{0} \equiv 0$ and if $0 \in X_{r}$ for only a finite set of $r$ values 
with $b>0$, it follows from the properties of exponentials and the boundedness of $\left\|E_{r}\right\|_{x}$, that $\left\{E_{r}(x)\right\}$ is bounded on $[\delta, 1-\delta], \delta>0$. Hence, $\left\{E_{r}(x)\right\}$ or a subsequence thereof converges pointwise to $E_{0}(x)$, an exponential, on $(0,1)$. From this reasoning, as before using varisolvence, $E_{0}$ is a best approximation to $f$ on $[0,1]$.

From the proof of Theorem 1 we have

COROLlARY. The sequence $\left\{E_{r}(x)\right\}$ has a subsequence which converges pointwise except possibly at the endpoints to a best exponential approximation, $E_{0}(x)$, to $f$ on $[0,1]$. If the subsequence converges to $E_{0}(0)$ and $E_{0}(1)$ also, then the convergence is uniform.

Department of Mathematics

Boston University

Boston, Massachusetts 02215

1. T. J. Rivlin \& E. W. ChENEY, "A comparison of uniform approximations on an interval and a finite subset thereof," SIAM J. Numer. Anal., v. 3, 1966, pp. 311-320. MR 34 \#4773.

2. C. B. Dunham, "Rational Chebyshev approximation on subsets," J. Approximation Theory, v. 1, 1968, pp. 484-487. MR 38 \#6279.

3. J. R. RICE, The Approximation of Functions. Vol. 2: Nonlinear and Multivariate Theory, Addison-Wesley, Reading, Mass., 1969. MR 39 \#5989. 